\title{
Electromagnetic Radiation from the Geometrical Point of View
}

\author{
Divakov Dmitriy ${ }^{1}$, Malykh Mikhail ${ }^{1}$, and Tiutiunnik Anastasiia ${ }^{1}$ \\ ${ }^{1}$ Peoples' Friendship University of Russia (RUDN University), 6 Miklukho-Maklaya St, Moscow, 117198, Russian Federation
}

\begin{abstract}
The article describes the relationship between the solutions of Maxwell's equations which can be considered at least locally as plane waves and the curvilinear coordinates of geometrical optics. We introduce phase-ray coordinate system for any electromagnetic field if vectors $\mathbf{E}$ and $\mathbf{H}$ are orthogonal to each other and their directions do not change with time $t$, but may vary from point to point in the domain $G$.
\end{abstract}

\section{Introduction}

Assuming that in the domain $(x, y, z) \in G \subset \square^{3}, \quad t>0$ with isotropic filling $\varepsilon$ and $\mu$ two vector fields $\mathbf{E}$ and $\mathbf{H}$ are given, satisfying the homogeneous system of Maxwell's equations

$$
\operatorname{rot} \mathbf{E}=-\frac{\mu}{c} \frac{\partial \mathbf{H}}{\partial t}, \operatorname{rot} \mathbf{H}=\frac{\varepsilon}{c} \frac{\partial \mathbf{E}}{\partial t} .
$$

It is well known that there are solutions of the system, which can be described by means of geometrical optics. These are, e.g., the plane waves in a homogeneous medium. Under the traditional approach it would be expected that in the general case, the laws of geometrical optics are valid only for harmonic vibrations, and only in the high-frequency limit. But yet Lüneburg [1] noted that the movement of discontinuity surfaces of electromagnetic fields obey the laws of geometrical optics exactly, and not only in the high-frequency limit. In our researches [2] we try to introduce the concepts of rays and phase fronts for solving Maxwell's equations without reference to the high-frequency limit and prove that the so-imposed rays obey the laws of geometrical optics.

\section{Phase-ray coordinate system}

For electromagnetic fields which can be considered at least locally as plane waves, vectors $\mathbf{E}$ and $\mathbf{H}$ are orthogonal to each other and their directions do not change with time $t$, but may vary from point to point in the domain $G$, as it occurs, for example, in the case of spherical wave. Therefore, as the first condition for the considered electromagnetic field, assume the following. Let the vectors $\mathbf{e}_{2}= \pm \mathbf{E} /\|\mathbf{E}\|$ and $\mathbf{e}_{3}= \pm \mathbf{H} /\|\mathbf{H}\|$ do not depend on $t$ and are orthogonal to each other. The sign is chosen once in an arbitrary manner under assumption that $\mathbf{e}_{2}$ and $\mathbf{e}_{3}$ are smooth vector fields in the domain $G$. The integral curves of the vector field $\mathbf{S}=\mathbf{E} \times \mathbf{H}$ we will call the rays of the electromagnetic field $\{\mathbf{E}, \mathbf{H}\}$. The surface orthogonal to $\mathbf{S}=\mathbf{E} \times \mathbf{H}$ at all points, will be called the phase front of the electromagnetic field $\{\mathbf{E}, \mathbf{H}\}$.

By the assumption directions of vectors $\mathbf{E}$ and $\mathbf{H}$ do not change with time, so the vector $\mathbf{e}_{1}= \pm \mathbf{S} /\|\mathbf{S}\|$ does not depend on $t$ as well. The ray emanating from the point $\left(x_{0}, y_{0}, z_{0}\right)$, is a trajectory of a point which motion is described by the initial problem

$$
\dot{\mathbf{r}}=\mathbf{e}_{1}(\mathbf{r}),\left.\quad \mathbf{r}\right|_{t=0}=\left(x_{0}, y_{0}, z_{0}\right)^{T} .
$$

The existence and uniqueness of its solution follow directly from Cauchy's theorem. Therefore, no matter how complicated the considered electromagnetic field is, from any point $\left(x_{0}, y_{0}, z_{0}\right)$ it is always possible to emit a ray and it will be the only one.

Subject to Jacobi theorem the necessary and sufficient condition of holonomicity of field $\mathbf{A}$ is the equality $(\operatorname{rot} \mathbf{A}, \mathbf{A})=0$; scalar $(\operatorname{rot} \mathbf{A}, \mathbf{A})$ is called the quantity of field A nonholonomicity. Therefore, if the electromagnetic field satisfies Maxwell's equations (1) and the stated above conditions, by virtue of the theorems of Cauchy and Jacobi there are well defined: 1) two-parameter family of rays, and 2) one-parameter family of phase surfaces.

If the fields $\mathbf{E}$ and $\mathbf{H}$ satisfy the Maxwell's equations (1) and the stated above conditions, they are holonomic [2]. This theorem allows one to associate the field with a curvilinear orthogonal coordinate system in which the Maxwell's equations look particularly simple. Due to the theorem of Jacobi, there are three such functions $u_{1}, u_{2}$ and $u_{3}$, that

$$
\mathbf{S} \square \nabla u_{1}, \quad \mathbf{E} \square \nabla u_{2}, \quad \mathbf{H} \square \nabla u_{3} .
$$

These functions define an orthogonal curvilinear coordinate system, which will be called phase-ray coordinate system.

Let $h_{1}, h_{2}, h_{3}$ be the Lame coefficients of the coordinate system. Using the same method [3] that is used in the theory of diffraction when introducing Borgnis functions [4], we can prove [2] that the field can be represented as 


$$
\mathbf{E}=\frac{E}{h_{2}} \mathbf{e}_{2}, \mathbf{H}=\frac{H}{h_{3}} \mathbf{e}_{3}
$$

where scalars $E$ and $H$ satisfy the system

$$
\left\{\begin{array}{c}
\frac{\partial}{\partial x_{1}} E+m_{e} \frac{\partial}{\partial t} H=0 \\
\frac{\partial}{\partial x_{1}} H+m_{h} \frac{\partial}{\partial t} E=0 \\
\frac{\partial}{\partial x_{3}} E=0, \\
\frac{\partial}{\partial x_{2}} H=0
\end{array}\right.
$$

the coefficients of which $m_{e}$ and $m_{h}$ are expressed in terms of the characteristics of filling $\varepsilon$ and $\mu$ in the region and the Lame coefficients by the formulas

$$
m_{e}=\frac{\mu}{c} \frac{h_{1} h_{2}}{h_{3}}, m_{h}=\frac{\varepsilon}{c} \frac{h_{1} h_{3}}{h_{2}} .
$$

In general, the system (5) comprises only four equations and two unknown functions, so from general considerations one would expect that the condition of the solvability of this system provides some restrictions on the coefficients $m_{e}$ and $m_{h}$. For the system (5) has a non-static solution it is necessary that one of its coefficients $m_{e}$ and $m_{h}$ can be represented as a product of the form $\phi\left(x_{1}, x_{2}\right) \psi\left(x_{1}, x_{3}\right)$ and it is enough that both coefficients $m_{e}$ and $m_{h}$ depend only on $x_{1}$ [2].

Sufficient conditions immediately refer us to geometrical optics. By the hypothesis in the curvilinear coordinate system under consideration the value

$$
c^{2} m_{e} m_{h}=\varepsilon \mu h_{1}^{2}
$$

is a positive constant, say $a^{2}$. Let the points $A$ and $B$ lie on a coordinate curve $x_{1}=c_{1}$, then the optical length of the arc of any curve connecting these points is equal to the expression

$$
\int_{A}^{B} \sqrt{\varepsilon \mu} d s=\int_{x_{1}=a}^{b} \sqrt{a^{2}+\varepsilon \mu h_{2}^{2}\left(\frac{d x_{2}}{d x_{1}}\right)^{2}+\varepsilon \mu h_{3}^{2}\left(\frac{d x_{3}}{d x_{1}}\right)^{2}} d x_{1} .
$$

This value will be minimal, if we take as the curve the arc of coordinate curve when

$$
\int_{A}^{B} \sqrt{\varepsilon \mu} d s=\int_{x_{1}=a}^{b} \sqrt{\varepsilon \mu} h_{1} d x_{1} .
$$

Thus $x_{1}$-lines are rays of geometrical optics [2].

The wave propagation along the rays will be subject to the equations system (5). To investigate the type of this system let us excluded a variable $H$ from the system (5). To this end, we differentiate the first equation of the system (5) by $x_{1}$, then we have

$$
\frac{\partial^{2} E}{\partial x_{1}^{2}}-m_{e} m_{h} \frac{\partial^{2} E}{\partial t^{2}}=\frac{\partial \ln m_{e}}{\partial x_{1}} \frac{\partial E}{\partial x_{1}},
$$

which is different from the usual hyperbolic equation by an additional term on the right side. This allows us to count on the extension of the class of problems whose solution can still be described by a system of rays and phase fronts. Equation (10) has the form

$$
\frac{\partial^{2} u}{\partial x_{1}^{2}}-\frac{1}{c^{2}} \frac{\partial^{2} u}{\partial t^{2}}=a \frac{\partial u}{\partial x}
$$

thus, it is a classical equation of oscillations with an additional term $a \frac{\partial u}{\partial x_{1}}$, which in the theory of oscillations is used for modeling damping vibrations phenomena due to the resistance of the medium [5]. If we take $a=0$ we get the usual equation of vibrations, so when choosing a certain initial conditions the instantaneous profile $u$ will move along the $x_{1}$-axis at the velocity $c$. Posing $a>0$ will lead to damping.

\section{The case of constant coefficients}

Using constants $m_{e}$ and $m_{h}$ the general solution of the system (5) can be written explicitly. Indeed, we differentiate the first equation (5) by $x_{1}$, then

$$
\frac{\partial^{2}}{\partial x_{1}^{2}} E+m_{e} \frac{\partial^{2}}{\partial x_{1} \partial t} H=0
$$

Using the second equation, we get

$$
\frac{\partial^{2}}{\partial x_{1}^{2}} E-m_{e} m_{h} \frac{\partial^{2}}{\partial t} E=0
$$

Classical electrodynamics considers media with positive $\varepsilon$ and $\mu$, Lame coefficients can not be negative as well. Therefore $m_{e} m_{h}>0$ and this equation is the hyperbolic equation, the general solution of which is given by d'Alembert's formula

$$
E=F_{1}\left(\sqrt{m_{e} m_{h}} x_{1}-t, x_{2}, x_{3}\right)+F_{2}\left(\sqrt{m_{e} m_{h}} x_{1}+t, x_{2}, x_{3}\right) \text {. }
$$

The third equation of the system indicates that these functions should not depend on $x_{3}$. Differentiating the fourth equation of the system with respect to $t$ and using the first equation, we get $\frac{\partial^{2} E}{\partial x_{1} \partial x_{2}}=0$. Therefore

$$
E=F_{1}\left(\sqrt{m_{e} m_{h}} x_{1}-t\right)+F_{2}\left(\sqrt{m_{e} m_{h}} x_{1}+t\right)+F_{3}\left(x_{2}\right) .
$$

We can write out a similar formula for $H$. Thus, in this case, the field is a superposition of two waves traveling along the rays in opposite directions, to which the static summand is added. The presence of this summand is due to the fact that earlier we have not excluded static fields from the consideration. Obviously when considering the electromagnetic radiation it is reasonable to omit it.

The $x_{1}$-lines of the phase-ray coordinate system represent rays of geometrical optics, and the field itself is a superposition of two waves running along the rays in opposite directions:

$$
\mathbf{E}=F\left(\sqrt{\varepsilon \mu} h_{1} x_{1}-c t\right) \frac{\mathbf{e}_{2}}{h_{2}}+G\left(\sqrt{\varepsilon \mu} h_{1} x_{1}+c t\right) \frac{\mathbf{e}_{2}}{h_{2}} .
$$

In particular, if the field in question has a gap at $t=0$, the gap propagates along the rays according to the laws of geometrical optics, as it should be, according to the Lüneburg [1]. Thus our approach give a true geometrical interpretation of Maxwell's equations, this is 
the first step on the way of the geometrization of classical electrodynamics [6-8]

\section{Hilbert-Courant waves}

Wishing to give a mathematical description of wave concepts, Hilbert and Courant [9] noticed that for hyperbolic equation

$$
\mathfrak{L}[u]=0
$$

one can specify such functions $\theta$ that for any choice of the function $W$ of one variable the expression

$$
u=W(\theta(\vec{x}, t))
$$

is the solution to the equation $\mathfrak{L}[u]$. These solutions can be interpreted as waves, the function $W$ - as a waveform, and $\theta$ - as its phase; the form of these waves is not distorted during propagation. At the same time it can be shown that the phase necessarily satisfies the eikonal equation. This concept can be generalized to the case of distorted waves, by considering the family of solutions

$$
\left\{u=g(\vec{x}, t) W(\theta(\vec{x}, t)) \quad \forall W \in C^{1}(\square)\right\} .
$$

The function $g$ introduced here, characterizes the distortion of the wave.

Localized wave solutions to the homogeneous scalar wave equation in free space are of three types: focus wave modes, superluminal and subluminal solutions. Each of them includes a subclass of solutions conforming to the Courant-Hilbert approach. One more class of solutions, spatiotemporally localized luminal null electromagnetic fields [10] also concern the Courant-Hilbert approach. They are transverse with respect to the local flow of energy. A class of luminal null electromagnetic waves has vortex structures on the planes transverse to the direction of propagation.

Null solutions of the Maxwell's equations were studied by Robinson in connection with his work on general relativity. A family of such null solutions of the Maxwell's equations was described, they are usually expressed in a special coordinate system.

There is a solution to the Maxwell's equations linked to a beam of light whose electric (magnetic) field lines are all closed loops with any two electric (magnetic) field lines linked to each other. Maxwell's equations preserve for such solutions the topological structure of the field lines. In [11] it was shown that such an evolution of the field is due to the condition that the electric and magnetic fields are everywhere orthogonal. For the wave equation one can find a variety of such waves [12].

As a part of our method Hilbert-Courant approach can be transferred to the vector case.

Non-permanent solution of (5)

$$
\begin{aligned}
& E=E\left(\theta\left(x_{1}, t\right)\right), \\
& H=H\left(\theta\left(x_{1}, t\right)\right)
\end{aligned}
$$

will be called the wave propagating along the ray without distortion, the function $\theta\left(x_{1}, t\right)$ will be called the phase of the wave.

The phase of any wave satisfies the eikonal equation

$$
\left(\frac{\partial \theta}{\partial x_{1}}\right)^{2}-\frac{\varepsilon \mu h_{1}^{2}}{c^{2}}\left(\frac{\partial \theta}{\partial t}\right)^{2}=0 .
$$

If the product $m_{e} m_{h}$ depends only on $x_{1}$, and the ratio $m_{e} / m_{h}$ is a constant, then any non-permanent solution of the eikonal equation can be in the form of a wave

$$
\begin{aligned}
& E=W\left(\theta\left(x_{1}, t\right)\right), \\
& H= \pm \sqrt{\frac{m_{h}}{m_{e}}} W\left(\theta\left(x_{1}, t\right)\right) \quad \forall W \in C^{1}(\square),
\end{aligned}
$$

propagating along the ray without distortion.

The transition from auxiliary functions $E, H$ to the electromagnetic field by the formulas (4) introduces additional factors which depend on the Lame coefficients. Therefore, the solution of the eikonal equation can be in the form of a wave propagating along the ray with a certain distortion of the form due to curvature of rays.

\section{Monochromatic field at high frequencies}

Assuming that the solution (10) depends on time as $E=u\left(x_{1}\right) \sin \omega t$, and assuming for brevity $x_{1}=x$, we obtain

$$
\frac{d^{2} u}{d x^{2}}-\frac{1}{m_{e}} \frac{\partial m_{e}}{\partial x} \frac{d u}{d x^{2}}+\omega^{2} m_{e} m_{h} u=0 .
$$

In the high-frequency limit $\omega \rightarrow \infty$ one can try to find a solution by the JWKB method. Let us consider a solution as a series

$$
u=\exp \left(\frac{1}{\lambda} \int_{0}^{x} d x\left(u_{0}+\lambda u_{1}+\ldots\right)\right)
$$

in powers of a small parameter $\lambda=1 / \omega$. Thus, at high frequencies, any monochromatic solution of the auxiliary equation (10) is a superposition of scalar waves of the form

$$
\sqrt[4]{\frac{m_{e}}{m_{h}}} \sin \left(t \pm i \omega \int \sqrt{m_{e} m_{h}} d x+\text { const }\right),
$$

traveling along the ray in two directions. The dependence of $\sqrt[4]{m_{e} / m_{h}}$ on $x_{1}$ characterizes the change in the amplitude of these waves along the ray.

Let us verify the formula (25) by numerical experiments. Figures 1,2 shows the solutions of the equation (23) with $\omega=10$ for two types of filling $m_{e}=1 /\left(1+x^{2}\right), m_{h}=1$ and $m_{e}=1+x, m_{h}=1$, found by the Runge-Kutta method, the initial data are chosen in a way to achieve the best coincidence with the asymptote $x=0$. The graphs clearly show that the formula (25) gives the right course of solving the equation. 


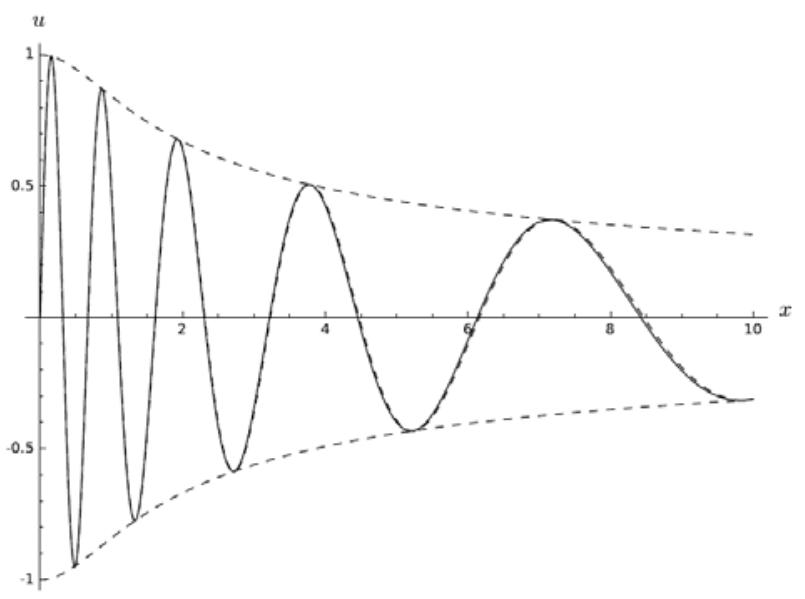

Fig.1. Solutions of the equation (23) and his asymptotics at $\omega=10$ for $m_{e}=1 /\left(1+x^{2}\right), m_{h}=1$.

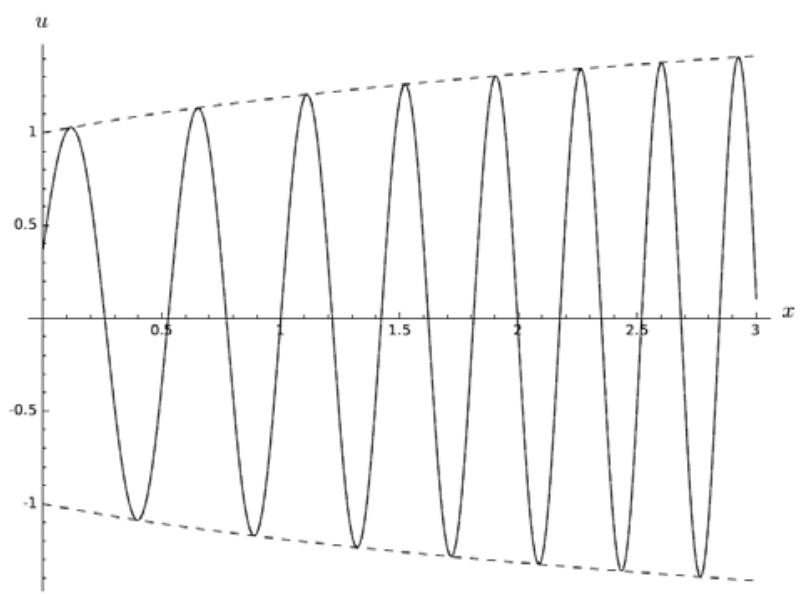

Fig.2. Solutions of the equation (23) and his asymptotics at $\omega=10$ for $m_{e}=1+x, m_{h}=1$.

\section{Conclusion}

On the assumption that in the region $G$ there is a homogeneous solution of Maxwell's equations, satisfying additional conditions of holonomicity of the Poynting vector field we build locally-orthogonal coordinate system (called the phase-ray system) having a clear geometric-optical interpretation. It is shown that under certain non-burdensome (sufficient) conditions on variable real coefficients of medium filling in the region (on the permittivity and magnetic susceptibility), a phase-ray coordinate system that describes the laws of geometrical optics in this region, can be constructed, and there exist solutions of Maxwell's equations, which take in this coordinate system the simplest (canonical) form.
The publication has been prepared with the support of the "RUDN University Program 5-100".

\section{References}

1. R. Lüneburg, Mathematical theory of optics. Providence (Rhode Island: Brown University, 1944)

2. M. D. Malykh, N. E. Nikolaev, L. A. Sevastianov, The Geometrical Description of Electromagnetic Radiation. Journal of Electromagnetic Waves and Applications 30(15), 2055-2066 (2017)

3. M. D. Malykh, On solutions of the Maxwell's equations from the viewpoint of geometrical optics. Bull PFUR, ser MIPh. 4, 65-72 (2014)

4. K. Zhang, D. Li, Electromagnetic theory for microwaves and optoelectronics (Berlin: Springer, 2007)

5. W. Yang, Reflection Seismology (Elsevier, 2014)

6. D.S. Kulyabov, A.V. Korolkova, L.A. Sevastianov, Spinor representation of Maxwell's equations. Journal of Physics: Conference Series. 788(1), 012025 (2017)

7. D.S. Kulyabov, A.V. Korolkova, L.A. Sevastianov, M.N. Gevorkyan, A.V. Demidova, Geometrization of Maxwell's equations in the construction of optical devices. Proceedings of SPIE - The International Society for Optical Engineering. 10337, 103370K (2017)

8. D.S. Kulyabov, A.V. Korolkova, L.A. Sevastianov, E.G. Eferina, T.R. Velieva, A geometric approach to the Lagrangian and Hamiltonian formalism of electrodynamics. Proceedings of SPIE - The International Society for Optical Engineering. 10337, 103370M (2017)

9. R. Courant, D. Hilbert, Methoden der mathematischen Physik (Springer Verlag, 1968)

10. I.M. Besieris, A.M. Shaarawi, Spatiotemporally localized null electromagnetic waves I. Luminal. Progress In Electromagnetics Research B 8, 1-28 (2008)

11. W.T.M. Irvine, Linked and knotted beams of light, conservation of helicity and the flow of null electromagnetic fields. J. Phys. A: Math. Theor. 43, 815-820 (2010)

12. I. M. Besieris, A. Shaarawi, Three classes of Courant-Hilbert progressive solutions to the scalar wave equation. Journal of electromagnetic waves and applications 16(8), 1047-1060 (2002) 